\title{
Overexpression of TAZ promotes cell proliferation, migration and epithelial-mesenchymal transition in ovarian cancer
}

\author{
GUANGYUAN CHEN, JIABIN XIE, PING HUANG and ZHIHONG YANG \\ Department of Gynecology, Songgang People's Hospital, Shenzhen, Guangdong 518105, P.R. China
}

Received March 31, 2015 ; Accepted May 5, 2016

DOI: $10.3892 / 01.2016 .4829$

\begin{abstract}
The Hippo pathway is dysregulated in multiple types of human cancer, including ovarian cancer. Nuclear expression of yes-associated protein 1 (YAP1), a downstream transcription coactivator of the Hippo pathway, has been demonstrated to promote tumorigenesis in ovarian cancer and may serve as a poor prognostic indicator. However, transcriptional coactivator with PDZ binding motif (TAZ), a downstream target of the Hippo pathway and paralog of YAP in mammalian cells, has not been fully investigated in ovarian cancer. The present study aimed to investigate the dysregulation and biological function of TAZ in ovarian cancer. Reverse transcription-quantitative polymerase chain reaction and western blotting revealed that TAZ mRNA and protein levels, respectively, were upregulated in ovarian cancer, and a meta-analysis of ovarian cancer microarray datasets identified that increased expression of TAZ mRNA is correlated with poor prognosis in patients with ovarian cancer. In addition, TAZ-knockdown in ovarian cancer cells demonstrated that TAZ regulates the migration, proliferation and epithelial-mesenchymal transition of ovarian cancer cells. Furthermore, pharmacological disruption of the YAP/TAZ/TEA domain protein complex resulted in a decrease in ovarian cancer cell migration, proliferation and vimentin expression. The results of the present study indicate that the overexpression of TAZ is important in the development and progression of ovarian cancer, and may function as a potential drug target for treatment of this disease entity.
\end{abstract}

\section{Introduction}

Ovarian cancer is the third most prevalent malignancy of the female reproductive system $(1,2)$. Of all the types of ovarian cancer, epithelial ovarian cancer has the poorest prognosis and is the primary cause of cancer-associated mortality in adult women $(1,2)$. Therefore, understanding the mechanisms that

Correspondence to: Dr Guangyuan Chen, Department of Gynecology, Songgang People's Hospital, 107 National Road Side Road, Shenzhen, Guangdong 518105, P.R. China

E-mail: gychendr@126.com

Key words: transcriptional coactivator with PDZ binding motif, Hippo pathway, verteporfin, ovarian cancer underlie the development and progression of ovarian cancer is the focus of numerous, intensive studies. Dysregulation of multiple signaling pathways has been implicated in the initiation, progression and metastasis of ovarian cancer, including the mitogen-activated protein kinase and phosphoinositide 3-kinase/AKT/mammalian target of rapamycin signaling pathways (2).

The Hippo pathway is an emerging signaling pathway that is crucial for tissue homeostasis, organ size control, cell differentiation and the development of various types of human cancer, including ovarian cancer $(3,4)$. The core components of the mammalian Hippo pathway consist of the upstream kinases Mst1/2, large tumor suppressor kinase 1 (LATS1/2) and their respective scaffold proteins, WW45 and MOB1. Activation of the Hippo tumor suppressor pathway increases the phosphorylation level of the transcription coactivator yes-associated protein 1 (YAP)/transcriptional coactivator with PDZ binding motif (TAZ), which results in the cytoplasmic retention of YAP/TAZ and protein degradation. As YAP/TAZ promotes cell proliferation and survival through the activation of downstream transcription factors, most notably the TEA domain (TEAD) transcription factor family members, activation of the Hippo pathway results in inhibition of TEAD-dependent transcription. Dysregulation of the Hippo pathway has been observed in human cancer, including ovarian cancer (5-9). The nuclear expression of YAP has been identified to promote ovarian cancer tumorigenesis and functions as a poor prognostic indicator for the disease (7). However, TAZ, a paralog of YAP in mammalian cells, has not yet been investigated in ovarian cancer.

The aim of the present study was to investigate the dysregulation and biological function of TAZ in ovarian cancer. The study identified that TAZ mRNA and protein are overexpressed in ovarian cancer, and a meta-analysis of an ovarian cancer database indicated that high TAZ mRNA expression correlated with poor prognosis in patients with ovarian cancer. In addition, TAZ-knockdown resulted in decreased proliferation and migration of ovarian cancer cells, and verteporfin, a compound that disrupts the interaction between YAP/TAZ and TEAD, decreased the viability of the ovarian cancer cells and almost abolished cell migration. Taken together, the results of the present study indicate that the overexpression of TAZ, a potential mechanism for the activation of YAP/TAZ downstream gene expression, may promote ovarian cancer tumorigenesis and progression, and may serve as a potential therapeutic drug target. 


\section{Materials and methods}

Ovarian cancer specimen collection. All human ovarian cancer and paired normal samples $(n=7)$ were obtained from the Department of Gynecology, Songgang People's Hospital, (Shenzhen, China) between December 2011 and February 2012. The cancer tissues and paired normal tissues were resected and fast frozen in liquid nitrogen, and then the samples were transferred into cryogenic tubes and stored at $-80^{\circ} \mathrm{C}$ for long-term storage. Informed consent was obtained from all patients and approval was obtained from the Ethics Committee of Songgang Hospital (Shenzhen, China) for the use of all specimens.

Cell culture and transfection. SKOV-3 ovarian cancer cells were obtained from the American Type Culture Collection (Manassas, VA, USA) and cultured in Dulbecco's modified Eagle's medium (Hyclone; GE Healthcare Life Sciences, Logan, UT, USA) supplemented with $10 \%$ fetal bovine serum (FBS; Sigma-Aldrich , St. Louis, MO, USA) and $1 \%$ penicillin/streptomycin at $37^{\circ} \mathrm{C}$ with $5 \% \mathrm{CO}_{2}$. Small interfering RNA (siRNA) transfection was performed using Lipofectamine ${ }^{\circledR} 2000$ (Invitrogen; Thermo Fisher Scientific, Inc., Waltham, MA, USA), according to the manufacturer's protocol. The TAZ siRNA primer sequences were as follows: TAZ-siRNA-1, GACAUG AGAUCCAUCACUA; and TAZ-siRNA-2, GGACAAACA CCCAUGAACA (10). TAZ siRNA and non-targeting control siRNA (UUCUCCGAACGUGUCACGU). were synthesized by Shanghai GenePharma Co., Ltd. (Shanghai, China).

$R N A$ isolation and reverse transcription-quantitative polymerase chain reaction (RT- $q P C R)$. Total RNA was extracted using TRIzol ${ }^{\circledR}$ reagent (Invitrogen; Thermo Fisher Scientific, Inc.) and reverse transcribed into cDNA with a PrimeScript ${ }^{\circledR}$ RT-PCR kit (Takara Biotechnology Co., Ltd., Dalian, China). qPCR was performed using SYBR ${ }^{\circledR}$ Premix Ex Taq ${ }^{\text {TM }}$ (Takara Biotechnology Co., Ltd.) on an ABI 7500 Real-Time PCR system (Applied Biosystems; Thermo Fisher Scientific, Inc.). The primers used were as follows: Forward, ATTCATCGC CTTCCTAGGGT and reverse, GGCTGGGAGATGACCTTC AC for TAZ; forward, GTCATCCAACGGGAATGCA and reverse, TGATCGGTTACCGTGATCAAAA for GAPDH. The cycling conditions were as follows: $95^{\circ} \mathrm{C}$ for $30 \mathrm{sec}$, followed by 40 cycles of $95^{\circ} \mathrm{C}$ for $5 \mathrm{sec}$ and $60^{\circ} \mathrm{C}$ for $34 \mathrm{sec}$. $\mathrm{ddH}_{2} \mathrm{O}$ was used as a negative control. The $2^{-\Delta \Delta C q}$ method was used for quantification (11).

Western blot analysis. Cancer tissues and cultured SKOV-3 cells were harvested and lysed with 1\% NP-40 lysis buffer [50 mM Tris-HCl, pH 7.8; $150 \mathrm{mM} \mathrm{NaCl;} 1 \%$ NP-40 with protease inhibitor cocktail (P8340; Sigma-Aldrich); phenylmethylsulfonyl fluoride; $50 \mathrm{mM} \mathrm{NaF}$; and $1 \mathrm{mM} \mathrm{Na} \mathrm{VO}_{4}$ ]. Protein (50 $\mu \mathrm{g}$ per sample) was separated by $10 \%$ SDS-PAGE and transferred to a nitrocellulose membrane. The membrane was blocked with $5 \%$ milk and incubated at $4^{\circ} \mathrm{C}$ overnight with the following primary antibodies: Monoclonal rabbit anti-YAP/TAZ (\#8418), monoclonal rabbit anti-zonula occludens-1 (ZO-1; \#8193) (Cell Signaling Technology, Inc., Danvers, MA, USA), monoclonal rabbit anti-vimentin (\#2707-1; Epitomics, Burlingame, CA, USA), monoclonal mouse E-cadherin (\#610181; BD Transduction Laboratories; BD Biosciences, Franklin Lakes, NJ, USA), monoclonal mouse N-cadherin (\#610920; BD Transduction Laboratories; BD Biosciences) and monoclonal mouse- $\beta$-actin (\#A1978; Sigma-Aldrich). All antibodies used in this study were diluted at a 1:1,000 ratio unless otherwise stated. Subsequently, the membrane was incubated with secondary antibodies horeradish peroxidase (HRP)-labeled goat anti-rabbit immunoglobulin (Ig) $\mathrm{G}(\mathrm{H}+\mathrm{L})$ \#A0208; Beyotime Institute of Biotechnology, Haimen, China) and HRP-labeled goat anti-mouse $\mathrm{IgG}(\mathrm{H}+\mathrm{L})$ (\#A0216; Beyotime Institute of Biotechnology), and the proteins were visualized using enhanced chemiluminescent reagents (EMD Millipore, Billerica, MA, USA).

Migration assay. Transwell systems (24-well insert; Corning Incorporated, Corning, NY, USA) were used to analyze cell migratory ability. siRNA-transfected SKOV-3 cells were suspended in media containing $1 \%$ FBS, seeded at a density of $1.0 \times 10^{5}$ cells/well in the upper chamber and incubated at $37^{\circ} \mathrm{C}$ with $5 \% \mathrm{CO}_{2}$. Cells on the upper membrane surface were removed 12 or $24 \mathrm{~h}$ later using a cotton bud, whilst cells on the lower membrane were fixed in $4 \%$ formaldehyde, stained with crystal violet and counted under a phase-contrast microscope.

Cell proliferation and viability assay. siRNA-transfected SKOV-3 cells were seeded in a 96-well plate (2,000 cells/well), and the number of cells was measured daily using a Cell Counting Kit-8 (CCK-8) assay (Dojindo Molecular Technologies, Inc., Kumamoto, Japan) for 5 days. For the verteporfin (\#SML0534; Sigma-Aldrich) treatment experiments, 6,000 SKOV-3 cells were seeded per well and treated with the indicated concentrations of verteporfin $(0.1,0.3,1.0,3.0$ and $9.0 \mu \mathrm{M}$ ) for 1 day, incubated at $37^{\circ} \mathrm{C}$ with $5 \% \mathrm{CO}_{2}$. Cell viability was measured by CCK-8 assay (Dojindo Molecular Technologies, Inc.), according to the manufacturer's protocol.

Statistical analysis. Kaplan-Meier plots of the overall and progression-free survival time of patients with ovarian cancer stratified by TAZ mRNA expression level were constructed using KMplot (http://kmplot.com/analysis). Survival data were analyzed using the log-rank test; all other data were analyzed using Student's t-test. Results are presented as the mean \pm standard deviation. Statistical analysis was performed using SPSS 19.0 (IBM SPSS, Armonk, NY, USA). P $<0.05$ was considered to indicate a statistically significant difference. For the cell proliferation and Transwell assays, each experiment was repeated twice. Typical results of one experiment are shown. For the cell proliferation assay, standard deviation was calculated based on the use of 6 repeated wells per group. For the Transwell assay, standard deviation was calculated based on 3 repeated Transwells per group.

\section{Results}

TAZ expression is upregulated in ovarian cancer. TAZ is a paralog of YAP in mammalian cells $(3,4)$ and dysregulation of YAP has been reported in ovarian cancer; however, the function of TAZ in ovarian cancer has not yet been investigated. To understand the dysregulation of TAZ in ovarian cancer, the expression of TAZ mRNA was analyzed in 7 ovarian cancer 


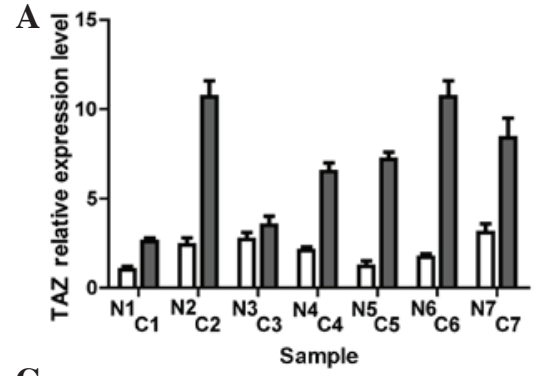

C

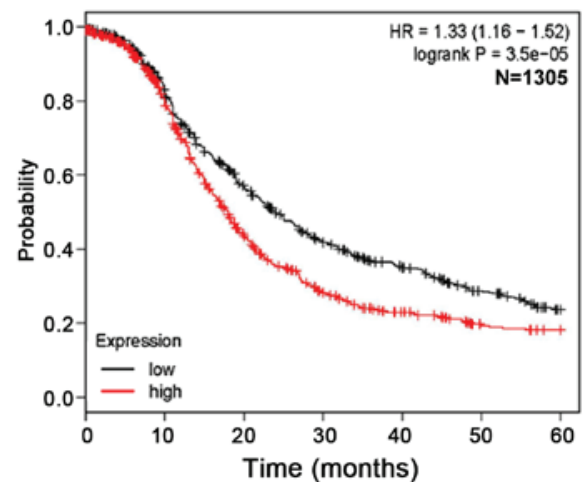

B

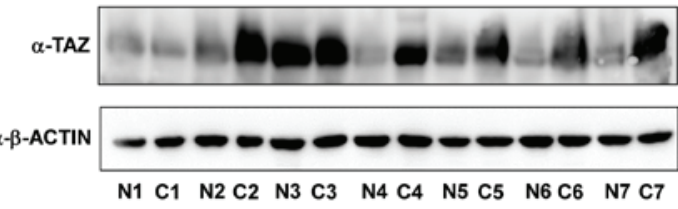

D

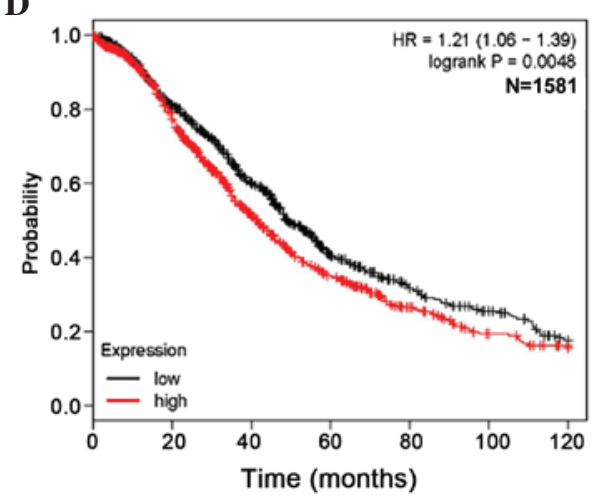

Figure 1. TAZ is overexpressed in and correlated with a poor prognosis in patients with ovarian cancer. (A) Reverse transcription-quantitative polymerase chain reaction analysis of TAZ mRNA expression levels and (B) western blot analysis of TAZ protein expression levels in the 7 ovarian cancer samples and paired normal tissues. Kaplan-Meier plots of the (C) progression-free and (D) overall survival time of patients with ovarian cancer stratified by TAZ mRNA expression level, constructed using KMplot (http://kmplot.com/analysis). The log-rank test was performed to assess statistical significance of survival data. N, normal; C, cancer; TAZ, tafazzin; HR, hazard ratio.

A

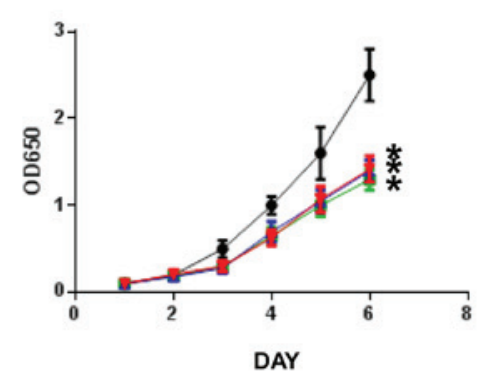

\section{B}

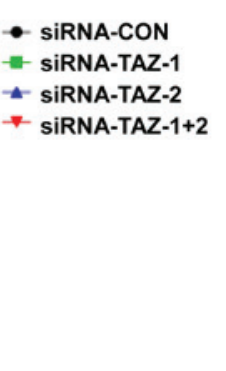

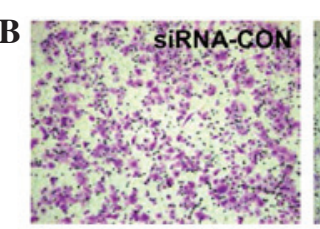
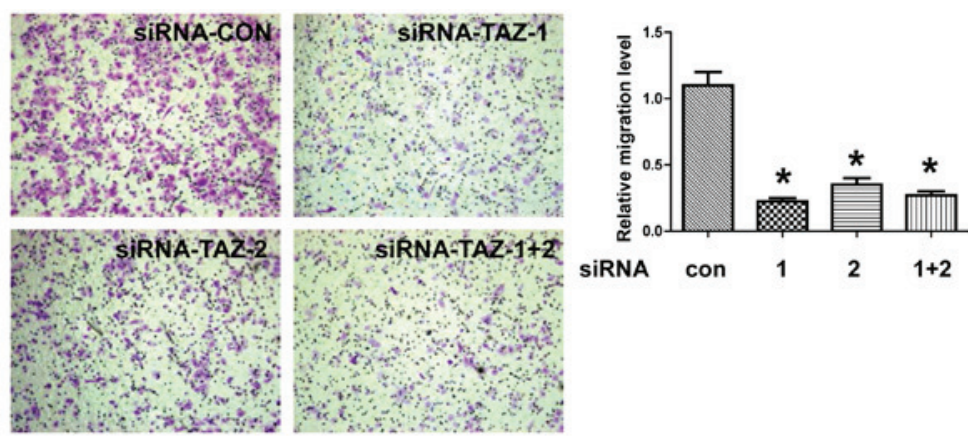

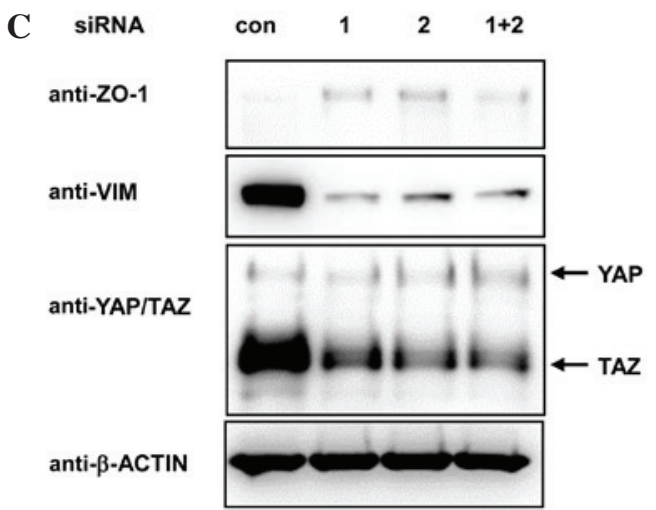

Figure 2. TAZ-knockdown inhibits cell proliferation and migration, and induces epithelial-mesenchymal transition in ovarian cancer cells. SKOV-3 cells were transfected with siRNA targeting human TAZ and control siRNA. (A) At $48 \mathrm{~h}$ post-transfection, the cells were plated into 96 -well plates and the cell numbers were detected by Cell Counting kit-8 assay. (B) Migratory ability of the SKOV-3 cells, as detected by Transwell assay. Representative images (x100 magnification) of each group are shown. Crystal violet staining. "P $<0.05$ vs. con, Student's t-test. (C) Western blot analysis of SKOV-3 cells transfected with indicated siRNA 72 h post-transfection. OD, optical density; siRNA, small interfering RNA; CON, control; TAZ, tafazzin; ZO, zonula occludens; VIM, vimentin; YAP, yes-associated protein 1 .

and paired normal tissue samples. Notably, as presented in Fig. 1A, TAZ mRNA was upregulated in 6/7 ovarian cancer samples compared with the paired normal tissues. Western blot analysis of these 7 paired cancer and normal tissues also 
showed that TAZ protein was overexpressed in $5 / 7$ ovarian cancer tissues (Fig. 1B). These results suggest that TAZ mRNA and protein expression are upregulated in ovarian cancer. To investigate the association between upregulated TAZ expression and the prognosis of patients with ovarian cancer, the present study analyzed a public online database (12) integrating 13 public datasets with 1,305 cases with progression-free data and 1,581 cases with overall survival data, and observed that high expression of TAZ mRNA was a significant indicator of poor prognosis in ovarian cancer. Patients with a high expression level of TAZ mRNA had a shorter period of progression-free $(\mathrm{P}=0.000035$; Fig. 1C) and overall survival ( $\mathrm{P}=0.0048$; Fig. 1D).

$T A Z$ regulates cell proliferation, migration and epithelial-mesenchymal transition (EMT) in ovarian cancer cells. To investigate the function of TAZ in ovarian cancer cells, two siRNAs targeting human TAZ were transfected into SKOV-3 cells and cell proliferation was analyzed following transfection. Transfecting each TAZ siRNA either individually or in combination resulted in a significant decrease in the proliferation of the SKOV-3 cells $(\mathrm{P}=0.00004$, siRNA-TAZ-1+2 vs. siRNA-CON; Fig. 2A). Next, the current study performed Transwell assays to determine whether TAZ-knockdown affects the migratory ability of ovarian cancer cells. As presented in Fig. 2B, knockdown of TAZ in the SKOV-3 cells largely decreased their migratory ability compared with control cells $(\mathrm{P}=0.000089)$. Overexpression of TAZ in breast cancer promotes EMT $(13,14)$, therefore, the protein expression levels of several EMT markers were also examined in the TAZ-knockdown cancer cells. Although no changes were observed in canonical EMT markers, including E-cadherin and $\mathrm{N}$-cadherin (data not shown), a moderate increase in the expression level of epithelial marker ZO-1 and a decrease in the level of mesenchymal marker vimentin were identified in TAZ-knockdown versus control cells (Fig. 2C). These results indicate that mesenchymal-epithelial transition was induced by TAZ-knockdown in the SKOV-3 cells. Taken together, the data suggests that TAZ serves a vital role in promoting proliferation, migration and EMT in ovarian cancer cells.

Pharmacological disruption of the interaction between YAP/TAZ and TEAD decreases cell viability and migration, and induces EMT in ovarian cancer cells. YAP/TAZ have been demonstrated to promote the tumorigenesis and progression of multiple types of cancer primarily through TEAD family members $(14,15)$, therefore, disrupting the interaction between YAP/TAZ and TEAD may serve as a promising target for new drugs. Verteporfin, a drug used previously for the treatment of macular degeneration, was identified to disrupt the YAP/TEAD complex $(16,17)$. The present study observed that verteporfin treatment results in decreased SKOV-3 cell viability $(\mathrm{P}=0.0014,3$ vs. $0 \mu \mathrm{M})$ Fig. 3A). Furthermore, verteporfin treatment significantly decreased and nearly abolished the migratory ability of the SKOV-3 cells ( $\mathrm{P}=0.0000043$; Fig. 3B). Similar to the effect of TAZ-knockdown on the SKOV-3 cells, the current study observed markedly decreased vimentin expression levels in the SKOV-3 cells following verteporfin treatment (Fig. 3C). These results indicate that disruption of the YAP/TAZ/TEAD
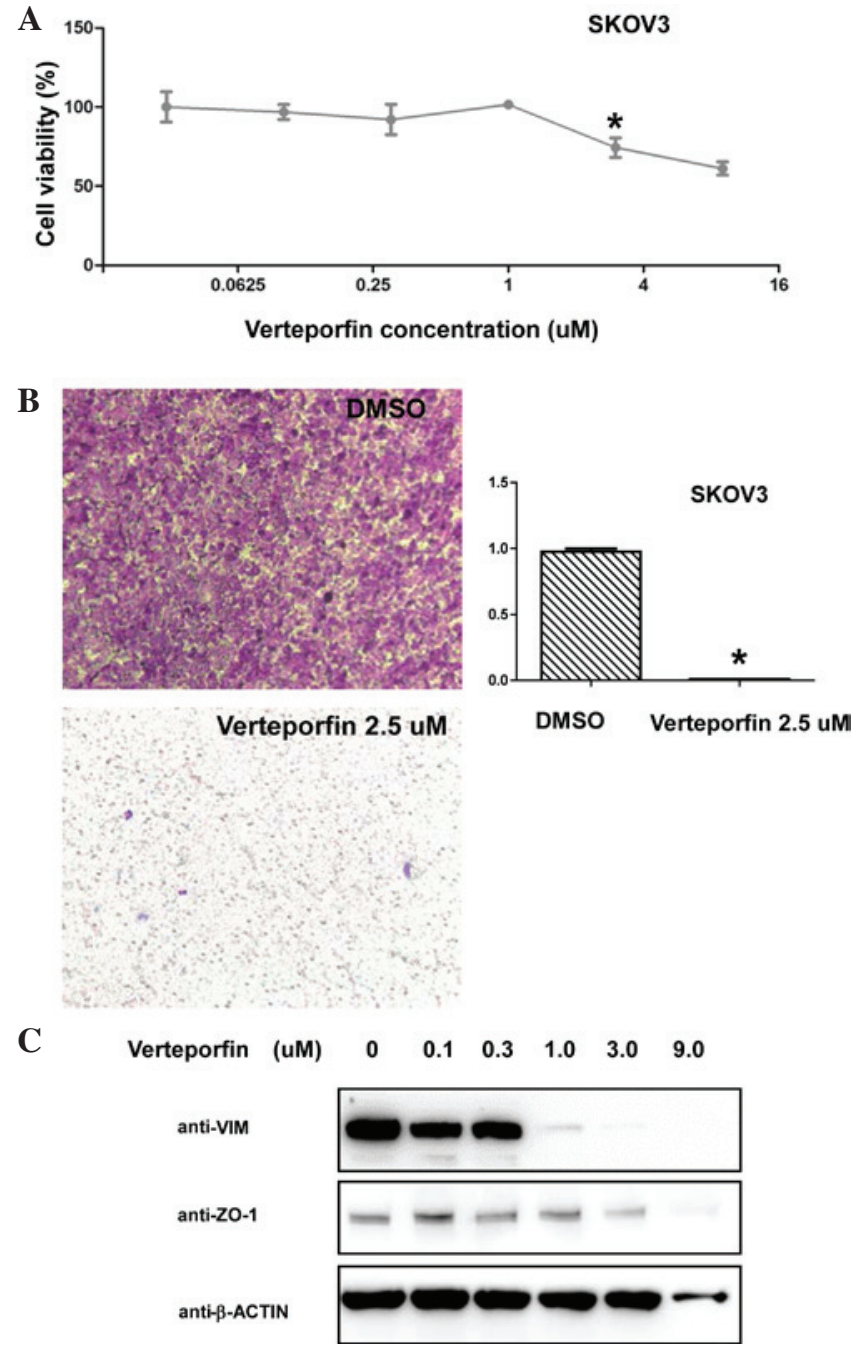

Figure 3. Pharmacological inhibition of taffazin/TEA domain interactions decreases cell viability and migration, and induces EMT in ovarian cancer cells. SKOV-3 cells were treated with the indicated concentrations of verteporfin for $24 \mathrm{~h}$ prior to analysis. (A) Cell viability was analyzed by Cell Counting kit- 8 assay. (B) Cell migration was analyzed by Transwell assay. Representative images (x100 magnification) of each group are shown. Crystal violet staining. ${ }^{*} \mathrm{P}<0.05$, Student's t-test. (C) Protein expression was analyzed by western blotting. DMSO, dimethyl sulfoxide; VIM, vimentin; ZO-1, zonula occludens-1.

complex mimics the effect of TAZ-knockdown in SKOV-3 ovarian cancer cells.

\section{Discussion}

Dysregulation of the Hippo tumor suppressor pathway has been observed in multiple types of human cancer. Inactivation of upstream tumor suppressors (including hypermethylation of Mst1 or decreased expression of LATS1/2) or activation of downstream oncogenes YAP/TAZ result in enhanced cell proliferation, inhibition of cell apoptosis and promotion of metastasis $(3,4)$. In ovarian cancer, nuclear expression of YAP, indicative of YAP activation, was identified to correlate with poor prognosis (7). Furthermore, the overexpression of Drosophila $\mathrm{Yki}^{\mathrm{S} 168 \mathrm{~A}}$ or human $\mathrm{YAP}^{\mathrm{S} 127 \mathrm{~A}}$, a constitutively active Yki/YAP mutant, was reported to induce tumorigenesis in the Drosophila ovary (5). Such data indicates the vital role of YAP/TAZ activation in ovarian cancer. 
TAZ, first identified as a 14-3-3 binding protein, shares $50 \%$ of its amino acid sequence with YAP in mammalian cells (18). Although the biochemical regulation of YAP/TAZ by the Hippo pathway is similar, the functions of YAP/TAZ are different in certain aspects. For example, in mice, Taz knockout leads to the development of polycystic kidney disease and emphysema (19), while Yap knockout results in embryonic lethality (20). The transcriptional regulation of YAP and TAZ also differ from one another. Gender determining region $\mathrm{Y}$ box 2 (21) and GA binding protein (22) have been reported to regulate YAP transcription; however, no transcription factor has yet been identified to regulate the transcription of TAZ. Although the TAZ gene locus amplification has been identified in $10 \%$ of ovarian cancer samples in The Cancer Genome Atlas datasets (23), further efforts are warranted to determine whether additional transcription factors are implicated in the overexpression of TAZ in ovarian cancer. In addition, despite similarities between the biochemical regulatory mechanisms and primary downstream target genes of YAP and TAZ, it was reported that YAP and TAZ also regulate different downstream target genes (14), which may result in the proteins exerting distinct functions in ovarian cancer development and progression. The identification of more TAZ downstream target genes in ovarian cancer may elucidate novel functions of the protein in the development of this disease.

Overexpression of TAZ and activation of YAP have been observed in ovarian cancer, therefore, TAZ/YAP may function as a potential drug target for the treatment of ovarian cancer. Furthermore, disruption of the YAP/TAZ/TEAD complex has been reported to inhibit YAP/TAZ-induced tumorigenesis in liver cancer models (16). The present study demonstrated that verteporfin treatment induced a similar phenotype to that observed following TAZ-knockdown in the SKOV-3 cells, further indicating that disruption of the YAP/TAZ/TEAD complex may function as a therapeutic target in patients with ovarian cancer.

In conclusion, the results of the current study indicate that overexpression of TAZ at the mRNA and protein level promotes the tumorigenesis and progression of ovarian cancer, and may, therefore, serve as a potential therapeutic drug target for this disease.

\section{Acknowledgements}

The present study was supported by the Key Specialized Research Funds of Songgang People's Hospital (Shenzhen, China).

\section{References}

1. Cannistra SA: Cancer of the ovary. N Engl J Med 351: 2519-2529, 2004.

2. Cho KR and Shih IeM: Ovarian cancer. Annu Rev Pathol 4 : 287-313, 2009

3. Plouffe SW, Hong AW and Guan KL: Disease implications of the Hippo/YAP pathway. Trends Mol Med 21: 212-222, 2015.

4. Harvey KF, Zhang X and Thomas DM: The Hippo pathway and human cancer. Nat Rev Cancer 13: 246-257, 2013.
5. Hall CA, Wang R, Miao J, Oliva E, Shen X, Wheeler T, Hilsenbeck SG, Orsulic S and Goode S: Hippo pathway effector Yap is an ovarian cancer oncogene. Cancer Res 70: 8517-8525, 2010.

6. Zhang X, George J, Deb S, Degoutin JL, Takano EA, Fox SB, Bowtell DD and Harvey KF; AOCS Study group: The Hippo pathway transcriptional co-activator, YAP, is an ovarian cancer oncogene. Oncogene 30: 2810-2822, 2011.

7. Xia Y, Chang T, Wang Y, Liu Y, Li W, Li M and Fan HY: YAP promotes ovarian cancer cell tumorigenesis and is indicative of a poor prognosis for ovarian cancer patients. PLoS One 9: e91770, 2014.

8. Xia Y, Zhang YL, Yu C, Chang T and Fan HY: YAP/TEAD co-activator regulated pluripotency and chemoresistance in ovarian cancer initiated cells. PLoS One 9: e109575, 2014

9. He C, Lv X, Hua G, Lele SM, Remmenga S, Dong J, Davis JS and Wang C: YAP forms autocrine loops with the ERBB pathway to regulate ovarian cancer initiation and progression. Oncogene 34: 6040-6054, 2015.

10. Calvo F, Ege N, Grande-Garcia A, Hooper S, Jenkins RP, Chaudhry SI, Harrington K, Williamson P, Moeendarbary E, Charras G and Sahai E: Mechanotransduction and YAP-dependent matrix remodelling is required for the generation and maintenance of cancer-associated fibroblasts. Nat Cell Biol 15: 637-646, 2013.

11. Livak KJ and Schmittgen TD: Analysis of relative gene expression data using real-time quantitative PCR and the 2(-Delta Delta C(T)) method. Methods 25: 402-408, 2001.

12. Gyorffy B, Lánczky A and Szállási Z: Implementing an online tool for genome-wide validation of survival-associated biomarkers in ovarian-cancer using microarray data from 1287 patients. Endocr Relat Cancer 19: 197-208, 2012

13. Lei QY, Zhang H, Zhao B, Zha ZY, Bai F, Pei XH, Zhao S, Xiong Y and Guan KL: TAZ promotes cell proliferation and epithelial-mesenchymal transition and is inhibited by the hippo pathway. Mol Cell Biol 28: 2426-2436, 2008.

14. Zhang H, Liu CY, Zha ZY, Zhao B, Yao J, Zhao S, Xiong Y, Lei QY and Guan KL: TEAD transcription factors mediate the function of TAZ in cell growth and epithelial-mesenchymal transition. J Biol Chem 284: 13355-13362, 2009.

15. Zhao B, Ye X, Yu J, Li L, Li W, Li S, Yu J, Lin JD, Wang CY, Chinnaiyan AM, et al: TEAD mediates YAP-dependent gene induction and growth control. Genes Dev 22: 1962-1971, 2008.

16. Brodowska K, Al-Moujahed A, Marmalidou A, Meyer Zu Horste M, Cichy J, Miller JW, Gragoudas E and Vavvas DG: The clinically used photosensitizer Verteporfin (VP) inhibits YAP-TEAD and human retinoblastoma cell growth in vitro without light activation. Exp Eye Res 124: 67-73, 2014.

17. Liu-Chittenden Y, Huang B, Shim JS, Chen Q, Lee SJ, Anders RA, Liu JO and Pan D: Genetic and pharmacological disruption of the TEAD-YAP complex suppresses the oncogenic activity of YAP. Genes Dev 26: 1300-1305, 2012.

18. Kanai F, Marignani PA, Sarbassova D, Yagi R, Hall RA Donowitz M, Hisaminato A, Fujiwara T, Ito Y, Cantley LC and Yaffe MB: TAZ: A novel transcriptional co-activator regulated by interactions with 14-3-3 and PDZ domain proteins. EMBO J 19: 6778-6791, 2000.

19. Morin-Kensicki EM, Boone BN, Howell M, Stonebraker JR, Teed J, Alb JG, Magnuson TR, O'Neal W and Milgram SL: Defects in yolk sac vasculogenesis, chorioallantoic fusion and embryonic axis elongation in mice with targeted disruption of Yap65. Mol Cell Biol 26: 77-87, 2006.

20. Hossain Z, Ali SM, Ko HL, Xu J, Ng CP, Guo K, Qi Z, Ponniah S, Hong W and Hunziker W: Glomerulocystic kidney disease in mice with a targeted inactivation of Wwtr1. Proc Natl Acad Sci USA 104: 1631-1636, 2007.

21. Seo E, Basu-Roy U, Gunaratne PH, Coarfa C, Lim DS, Basilico C and Mansukhani A: SOX2 regulates YAP1 to maintain stemness and determine cell fate in the osteo-adipo lineage. Cell Reports 3: 2075-2087, 2013.

22. Wu H, Xiao Y, Zhang S, Ji S, Wei L, Fan F, Geng J, Tian J, Sun X, Qin F, et al: The Ets transcription factor GABP is a component of the hippo pathway essential for growth and antioxidant defense. Cell Reports 3: 1663-1677, 2013.

23. Bell D, Berchuck A, Birrer M, Chien J, Cramer DW, Dao F, Dhir R, DiSaia P, Gabra H, Glenn P, et al; Cancer Genome Atlas Research Network: Integrated genomic analyses of ovarian carcinoma. Nature 474: 609-615, 2011. 